\title{
Knockdown of Long Non-Coding RNA RP11-445H22.4 Alleviates LPS-Induced Injuries by Regulation of MiR-301a in Osteoarthritis
}

\author{
Taitao Sun ${ }^{a}$ Jian Yu Liang Han ${ }^{c}$ Shuo Tian ${ }^{d}$ Bin Xua Xianbin Gong ${ }^{a}$ \\ Qiang Zhao ${ }^{\mathrm{a}}$ Yang Wange
}

\begin{abstract}
aDepartment of Orthopedics, Jining No.1 People's Hospital, Jining, b Department of Orthopedics, Heze Municipal Hospital, Heze, 'Department of Orthopedics, Affiliated Hospital of Jining Medical University, Jining, 'Shandong Polytechnic College, Jining, eDepartment of Orthopedics, China-Japan Union Hospital of Jilin University, Changchun, China
\end{abstract}

\section{Key Words}

LncRNA RP11-445H22.4 • MiR-301a • Cell viability • Apoptosis • Inflammatory injury • CXCR4

\begin{abstract}
Background/Aims: Several long non-coding RNAs (IncRNAs) play vital roles in osteoarthritis $(\mathrm{OA})$, whereas the role of IncRNA RP11-445H22.4 in OA remains unclear. The study aimed to investigate the effect of IncRNA RP11-445H22.4 on lipopolysaccharide (LPS)-induced cell viability, apoptosis and inflammatory injury of OA. Methods: The expression of RP11$445 \mathrm{H} 22.4$, miR-301a and CXCR4 in human cartilage ATDC5 cells were altered by transfection, and then cells were exposed to $5 \mu \mathrm{g} / \mathrm{ml}$ LPS for $12 \mathrm{~h}$. Then cell viability, apoptosis, apoptosisrelated factors and inflammatory cytokines were analyzed by CCK-8, flow cytometry, western blot, RT-qPCR and ELISA, respectively. Dual-luciferase reporter assay was performed to assess the binging sites of RP11-445H22.4 and miR-301a. The signal pathways of NF-KB and MAPK/ ERK were determined by western blot. Results: LPS reduced cell viability, increased apoptosis and stimulated release of IL-1 $\beta$, IL- 6 , IL-8 and TNF- $\alpha$. However, RP11-445H22.4 inhibition significantly rescued LPS-induced injuries by promoting cell viability, suppressing apoptosis and inflammatory cytokines secretions in ATDC5 cells. In addition, miR-301a directly bound to RP11-445H22.4, and suppression of miR-301a inversed the effects of RP11-445H22.4 inhibition. Furthermore, CXCR4 was a direct target of miR-301a, and CXCR4 silencing increased cell viability, decreased apoptosis and inflammatory cytokines secretions in LPS-treated ATDC5 cells. Besides, we found that CXCR4 silencing blocked LPS-activated NF-KB and MAPK/ERK pathways. Conclusions: The study indicated that IncRNA RP11-445H22.4-miR-301a-CXCR4 axis played an important role in cartilage ATDC5 cells and provided a theoretical basis of IncRNA RP11-445H22.4 in OA.
\end{abstract}

T. Sun and J. Yu contributed equally to this work. 


\section{Introduction}

Osteoarthritis $(\mathrm{OA})$ is a joint disease, that caused by destruction of articular cartilage and underlying bone $[1,2]$. The common symptoms are joint pain, tenderness, swelling, stiffness, limitation of motion and deformity [3]. According to epidemiological analysis, approximately 190 million people are affected OA worldwide, and the most proportion in the elderly [4]. The treatment of $\mathrm{OA}$ is well accepted to be joint reconstruction via arthroplasty or used anti-inflammatory drugs to relieve OA [5, 6]. However, several side effects are always existed in these therapeutic methods. Therefore, to find a new effective therapeutic strategy is necessary.

Long non-coding RNAs (lncRNAs) is a non-coding RNA, length of more than $200 \mathrm{nt}$, exist in eukaryotic cells, and which is highly conserved among different species of mammals [7, 8]. Recently, increasing evidences demonstrated that lncRNAs are key elements of pathologic and physiologic processes, and are vital regulators of inflammatory response [9, 10]. Previous study reported that cartilage injury-related lncRNA (lncRNA-CIR) was higher expressed in chondrocytes induced by interleukin-1(IL-1), and inhibited the formation of collagen and aggrecan in OA [11]. In additional, Zhang et al. [12] exposed that IncRNA ubiquitinfold modifier conjugating enzyme 1 (UFC1) enhanced chondrocyte cell proliferation and reduced apoptosis by targeting miR-34a in OA. However, whether IncRNA RP11-445H22.4 was related to pathogenesis of $\mathrm{OA}$ remains unclear.

LncRNA RP11-445H22.4 is a gene which located on human chromosome 20 with a length of 862 bp [13]. Recent study demonstrated that IncRNA RP11-445H22.4 was highly expressed in breast cancer cells, and it was related to the occurrence of tumor induced inflammatory response [13]. Furthermore, high expression lncRNA RP11-445H22.4 have been found in OA cartilage cells, and it might be associated with the pathogenesis of OA [14]. But, little is known about the function of IncRNA RP11-445H22.4 in OA and its potential targets for therapy.

In this study, we aimed to investigate the effect of lncRNA RP11-445H22.4 on lipopolysaccharide (LPS)-induced cell growth and inflammation injury of ATDC5 cells and the potential mechanism. Cell viability and apoptosis were examined by Cell Counting Kit8 (CCK-8) and flow cytometry. The relative expressions of apoptosis-associated factors and different inflammation cytokines were detected by western blot and enzyme-linked immunosorbent assay (ELISA). Dual-luciferase reporter assays were performed to validate the function of the predicted binging sites of IncRNA RP11-445H22.4 and miR-301a. The signal pathways of nuclear factor (NF)- $\kappa \mathrm{B}$ and mitogen-activated protein kinase (MAPK)/ extracellular regulated protein kinases (ERK) were analyzed by western blot. This current study will provide a theoretical basis for further study concerning OA diagnosis and treatment.

\section{Materials and Methods}

\section{Cell culture and treatment}

Human cartilage ATDC5 cells were purchased from the American Type Culture Collection (ATCC, Manassas, VA) and cultured in complete medium of Roswell Park Memorial Institute (RPMI)-1640 (Invitrogen, Grand Island, NY) supplemented with 10\% fetal bovine serum (FBS, Gibco, Carlsbad, CA) in a humidified incubator containing $5 \% \mathrm{CO}_{2}$ at $37^{\circ} \mathrm{C}$. All cells were maintained in growth medium in a $75 \mathrm{~cm}_{2}$ flask. Fresh medium was added to cells every three days until confluence was achieved. Cells were treated with LPS at different concentrations of $0,1,5$ and $10 \mu \mathrm{g} / \mathrm{ml}$ for $12 \mathrm{~h}$. Non-treated cells were taken as control group.

Cell transfection

To analyze the effects of IncRNA RP11-445H22.4 and C-X-C chemokine receptor type 4(CXCR4), the full-length of RP11-445H22.4 and CXCR4 sequences and short-hairpin RNA (shRNA) directed against RP11- 


\section{Cellular Physiology Cell Physiol Biochem 2018;45:832-843

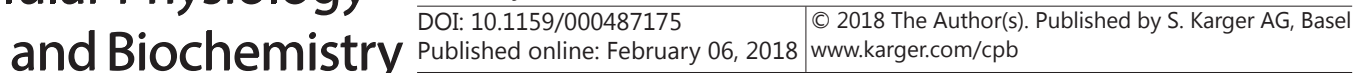 \\ Sun et al.: Role of LncRNA RP11-445H22.4 in OA}

445H22.4 and CXCR4 were constructed in pEX-2 and U6/GFP/Neo plasmids (GenePharma, Shanghai, China), respectively. They were referred as to pEX-RP11-445H22.4, pEX-CXCR4, sh-RP11-445H22.4 and sh-CXCR4. To analyze the effects of miR-301a, miR-301a mimic, miR-301a inhibitor and their respective controls were synthesized (Life Technologies Corporation, MD, USA) and transfected into ATDC5 cells in the in the study. All cell transfections were performed by using Lipofectamine 3000 reagent (Life Technologies Corporation) according to the manufacturer's instructions.

\section{Cell viability}

The ATDC5 cells of logarithmic growth phase were seeded in 96-well plate with a density of $1 \times 10^{4}$ cells/well, and the transfection was carried out when the cell fusion reached to $90 \%$. Then after transfection for $48 \mathrm{~h}$, the $10 \mu \mathrm{l}$ of CCK-8 (Dojindo Molecular Technologies, Gaithersburg, MD) solution was added to the culture medium, and incubated for $4 \mathrm{~h}$ in humidified atmosphere with $5 \% \mathrm{CO}_{2}$ at $37^{\circ} \mathrm{C}$. The optical density at $450 \mathrm{~nm}$ was analyzed by using a Microplate Reader (Bio-Rad, Hercules, CA).

\section{Apoptosis assay}

Cell apoptosis analysis was examined by Annexin V Apoptosis Detection Kit I (Univ-bio, Shanghai, China) according to the manufacturer's manual. In brief, ATDC5 cells $\left(1 \times 10^{5}\right)$ were washed twice with phosphate buffer saline (PBS), and then $10 \mu \mathrm{l}$ Annexin V-PE $(20 \mu \mathrm{g} / \mathrm{ml})$ was added to the solution, and all cells were incubated in the dark for $30 \mathrm{~min}$ at room temperature. Subsequently, $100 \mu \mathrm{l}$ Binging Buffer was added and the apoptotic cells were measured using a BD FACSCanto II flow cytometer (BD Biosciences, San Jose, CA).

\section{Real-Time quantitative PCR (RT-qPCR)}

Total RNA of ATDC5 cells were isolated by using Trizol reagent (Life Technologies Corporation) according to the manufacturer's instructions. Synthetic of complimentary DNA (cDNA) was performed by Transcriptor First Strand cDNA Synthesis Kit (Roche, USA). Real-Time PCR was conducted by using One Step SYBR® PrimeScript@PLUS RT-RNA PCR Kit (TaKaRa Biotechnology, Dalian, China). These data were normalized to GAPDH or U6 snRNA expression, and were texted by using the $2^{-\Delta \Delta C t}$ method.

\section{Luciferase reporter assay}

LncRNA RP11-445H22.4 and 3'-untranslated region (3'-UTR) of CXCR4 were amplified by PCR and then cloned into the pmiR-GLO dual-luciferase miRNA Target Expression Vector (Promega, Madison, WI, USA). Then the vectors were co-transfected with miR-301a mimic or mimic control into cells using Lipofectamine 3000 reagent (Life Technologies Corporation). After transfection for $48 \mathrm{~h}$, the Dual-Luciferase reporter assay system (Promega, Madison, WI, USA) was used for testing the luciferase activity.

\section{ELISA}

Culture supernatant was collected from 24-well plates and concentrations of inflammatory cytokines of interleukin-1 beta (IL-1 $\beta$ ), interleukin-6 (IL-6), interleukin-8 (IL-8), and tumor necrosis factor-alpha (TNF- $\alpha$ ) measured by ELISA using protocols supplied by the manufacturer (R\&D Systems, Abingdon, UK) and normalized to cell protein concentrations.

\section{Western blot}

Cellular protein was extracted using radio immunoprecipitation assay (RIPA) lysis buffer (Beyotime Biotechnology, Shanghai, China) supplemented with protease inhibitors (Roche, Basle, Switzerland). The protein concentration was determined by using the $\mathrm{BCA}^{\mathrm{TM}}$ Protein Assay Kit (Pierce, Appleton, WI, USA). Protein samples were separated by the sodium dodecyl sulfate-polyacrylamide gel electrophoresis (SDSPAGE) and transferred to nitrocellulose membranes (Maibio, Shanghai, China). After blocking with 5\% nonfat milk for $1 \mathrm{~h}$ at room temperature, the membranes were incubated with specific primary antibodies against B-cell lymphoma 2 (Bcl-2; ab32124), Bcl-2-associated X (Bax, ab32503), pro-caspase-3 (ab32150), cleaved caspase-3 (ab13585), pro-caspase-9 (ab32068), CXCR4 (ab1670), MARK (ab60202), c-Jun N-terminal kinase (JNK, ab208035), phosphorylated JNK (p-JNK, ab76572), c-Jun (ab119944), p-c-Jun (ab81319),

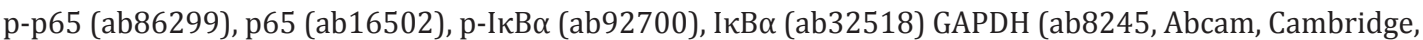
UK) and p-MARK (\#4836), cleaved caspase-9 (\#9501, Cell signaling Technology) (dilution 1:10, 00; Santa 


\section{Cellular Physiology Cell Physiol Biochem 2018;45:832-843

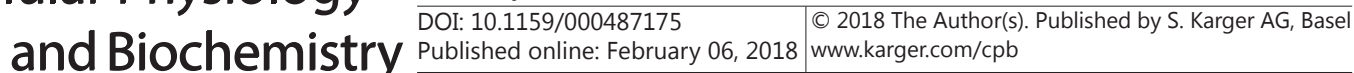 \\ Sun et al.: Role of LncRNA RP11-445H22.4 in OA}

Cruz Biotechnology, Santa Cruz, CA, USA) at $4{ }^{\circ} \mathrm{C}$ overnight, followed by wash and incubation with secondary antibody marked by horseradish peroxidase for $1 \mathrm{~h}$ at room temperature. The signals were captured by using Enhanced chemiluminescence (ECL) reagent (GE Healthcare, Little Chalfont, UK).

\section{Statistical analysis}

All experiments were repeated three times. The results of multiple experiments are presented as the mean \pm standard derivation (SD). Statistical analyses were performed using Graphpad 6.0 statistical software (GraphPad, San Diego, California, USA). The $P$-values were calculated using a one-way analysis of variance (ANOVA). A $P$-value of $<0.05$ was considered to indicate a statistically significant result.

\section{Results}

LPS reduced cell viability, induced apoptosis and inflammatory response in ATGC5 cells

To investigate the effect of LPS on OA, ATGC5 cells were treated with different concentrations of LPS $(0,1,5$ and $10 \mu \mathrm{g} / \mathrm{ml})$ for $12 \mathrm{~h}$. Then cell viability and apoptosis were analyzed by CCK8 and flow cytometry. As shown in Fig. 1A and 1B, LPS stimulation signally suppressed cell viability and promoted apoptosis at $5 \mu \mathrm{g} /$ $\mathrm{ml}$ and $10 \mu \mathrm{g} / \mathrm{ml}$ of LPS $(P<0.01$ or $P<0.001)$. Western blot results displayed that LPS induced the upregulation of Bax, cleaved-caspase-3 and cleaved-caspase- 9 but downregulation of Bcl-2. The expressions levels of pro-caspase- 3 and procaspase- 9 were not induced by LPS (Fig. 1C).

Next, RT-qPCR and ELISA were used to detect the different inflammatory cytokines expressions and secretions. RT-qPCR results showed that LPS enhanced IL-1 $\beta$, IL-6, IL-8 and TNF- $\alpha$ expression compared with control group (Fig. 1D, $P<0.05$ ). ELISA results showed consistent result that LPS remarkably stimulated release IL$1 \beta$, IL-6, IL-8 and TNF- $\alpha$ in ATGC5 cells (Fig. $1 \mathrm{E}, P<0.01$ or $P<0.001$ ). Taken together, these data indicated that LPS could reduce cell viability, increase apoptosis and stimulate release of different inflammatory cytokines in ATDC5 cells.

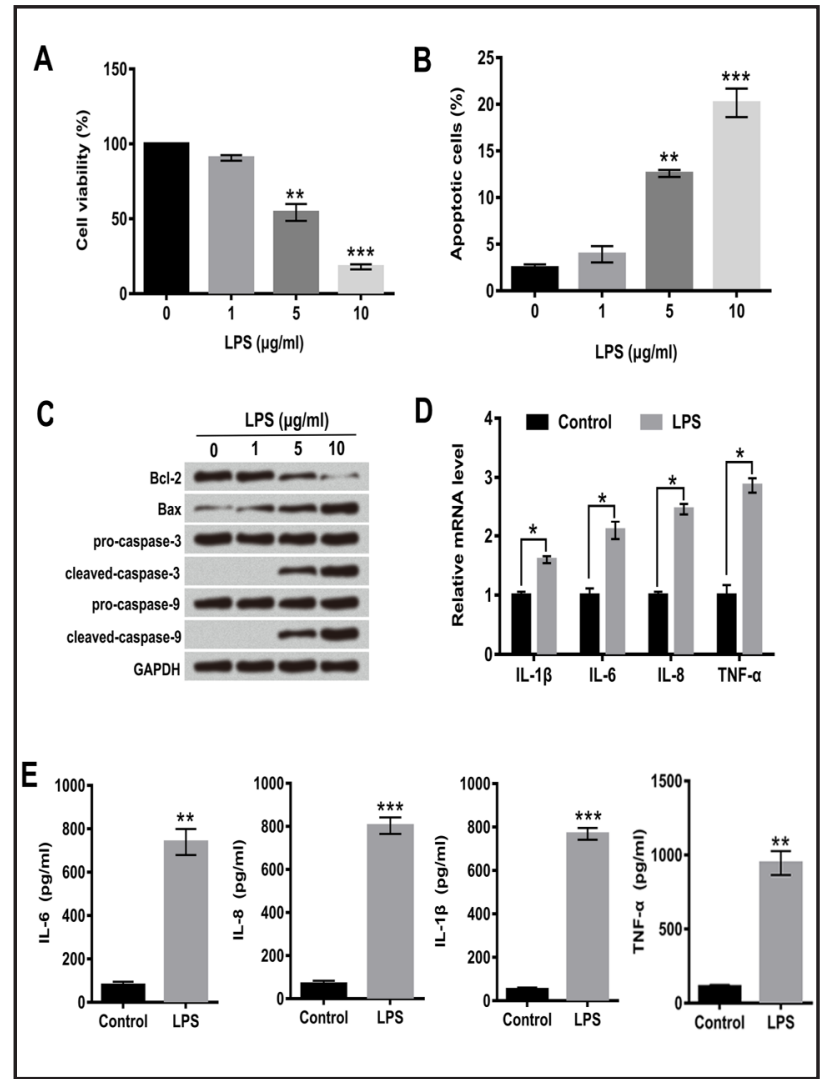

Fig. 1. LPS caused ATGC5 cells injuries by decreasing cell viability, increasing apoptosis and inducing inflammatory cytokines secretion. ATGC5 cells were treated by $0,1,5$, and 10 $\mu \mathrm{g} / \mathrm{ml} \mathrm{LPS}$ for $12 \mathrm{~h}$, and then (A) cell viability (B) cell apoptosis (C) apoptosis-related factor (D) mRNAs of IL-1 $\beta$, IL-6, IL-8 and TNF- $\alpha$ and (E) the concentrations of IL-1 $\beta$, IL- 6 , IL- 8 and TNF- $\alpha$ were detected by CCK-8, flow cytometry, western blot, RT-qPCR and ELISA. LPS: lipopolysaccharide; IL-1 $\beta$ : interleukin-1 beta; IL-6: interleukin-6; IL-8: interleukin-8; TNF- $\alpha$ : tumor necrosis factor-alpha; CCK-8: Cell Counting Kit-8; RTqPCR: real-time quantitative PCR; ELISA: enzyme linked immunosorbent assay; * $\mathrm{P}<0.05,{ }^{* *}, \mathrm{P}<0.01,{ }^{* * *}, \mathrm{P}<0.001$. 


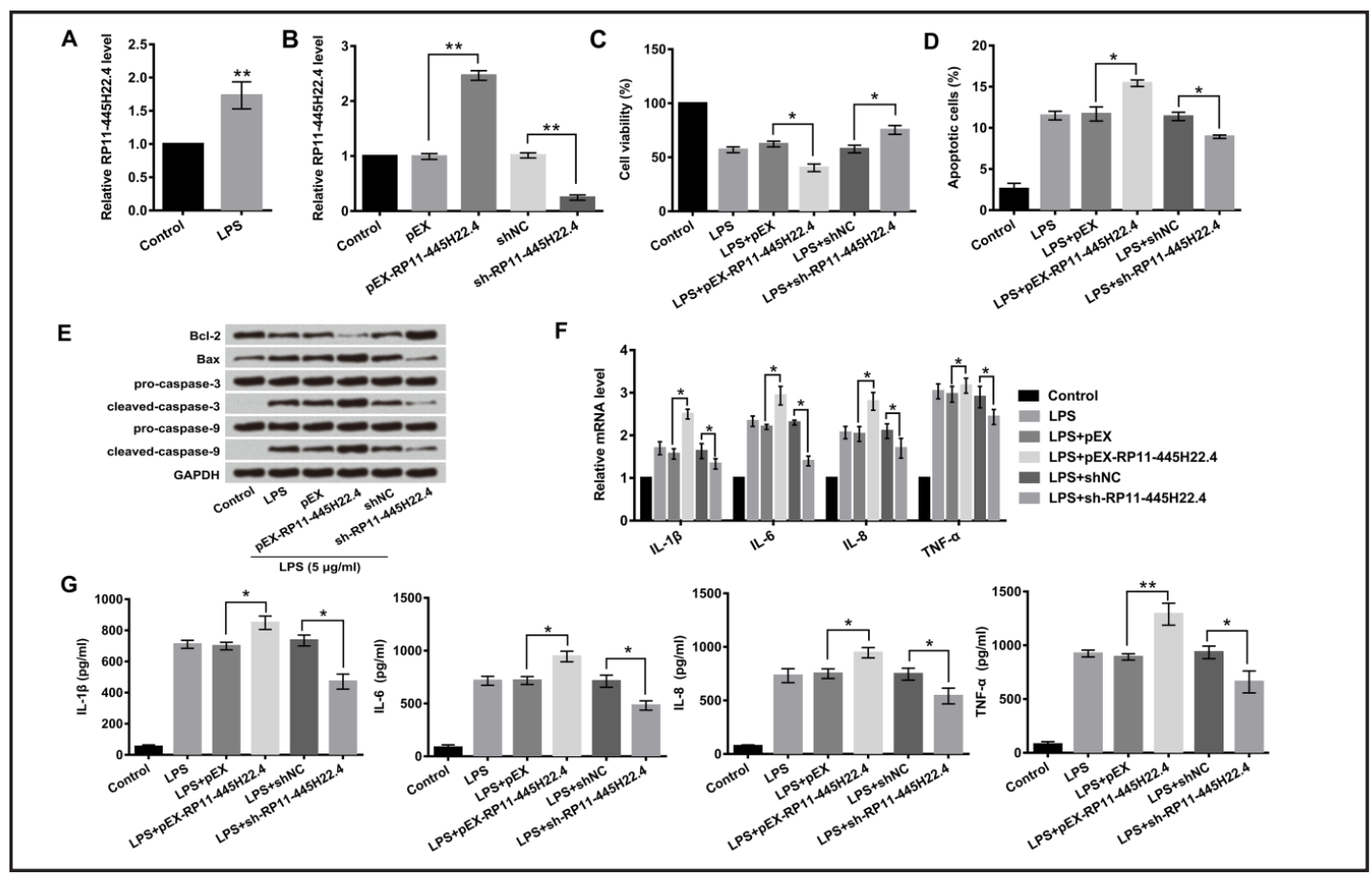

Fig. 2. LncRNA RP11-445H22.4 inhibition rescued LPS-induced cell injuries in ATGC5 cells. ATGC5 cells were transfected with pEX-RP11-445H22.4, sh-RP11-445H22.4 and corresponding controls. After stimulation by $5 \mu \mathrm{g} / \mathrm{ml}$ LPS, (A) relative expression of RP11-445H22.4 in LPS-induced cells and (B) relative expression of RP11-445H22.4 in RP11-445H22.4 overexpressed or suppressed cells were examined by RT-qPCR; (C) cell viability (D) cell apoptosis (E) protein levels of apoptosis-related factors (F) mRNA of inflammatory cytokines $(\mathrm{G})$ the concentration of inflammatory cytokines were detected by CCK-8, flow cytometry, western blot RT-qPCR, and ELISA, respectively. LPS: lipopolysaccharide; IncRNA: long non-coding RNA; RTqPCR: real-time quantitative PCR; CCK-8: Cell Counting Kit-8; ELISA: enzyme linked immunosorbent assay; $*, \mathrm{P}<0.05$, **, $\mathrm{P}<0.01$.

Fig. 3. LncRNA RP11-445H22.4 acted as a molecular sponge of miR-301a. ATGC5 cells were transfected with pEX-RP11445H22.4 and sh-RP11-445H22.4. (A) The expression level of RP11-445H22.4 was determined by RT-qPCR; (B) The activity of a luciferase reporter fused to RP11-445H22.4-Wt and RP11445H22.4-Mt were examined by dual-luciferase assay. MiR301a: microRNA-301a; lncRNA: long non-coding RNA; sh-RP11445H22.4: short hairpin-RP11-445H22.4; RT-qPCR: real-time quantitative PCR; Wt: Wild-type; Mt: Mutated-type; *, P<0.05.

LncRNA RP11-445H22.4 inhibition rescued LPSinduced cell injuries by promoting cell viability, suppressing apoptosis and inflammatory response

To explore the effect of lncRNA RP11-445H22.4 on LPS-induced cell injuries in OA, ATGC5 cells were transfected with pEX-RP11-445H22.4 and sh-RP11$445 \mathrm{H} 22.4$ to overexpress or suppress RP11-445H22.4 expression. Results showed that LPS significantly promoted RP11-445H22.4 expression (Fig. 2A, $P<0.01$ ). Relative expression of RP11-445H22.4 was up-regulated by pEX-RP11-445H22.4 while down-regulated by shRP11-445H22.4 (Fig. 2B, $P<0.01$ ). Cellular functional

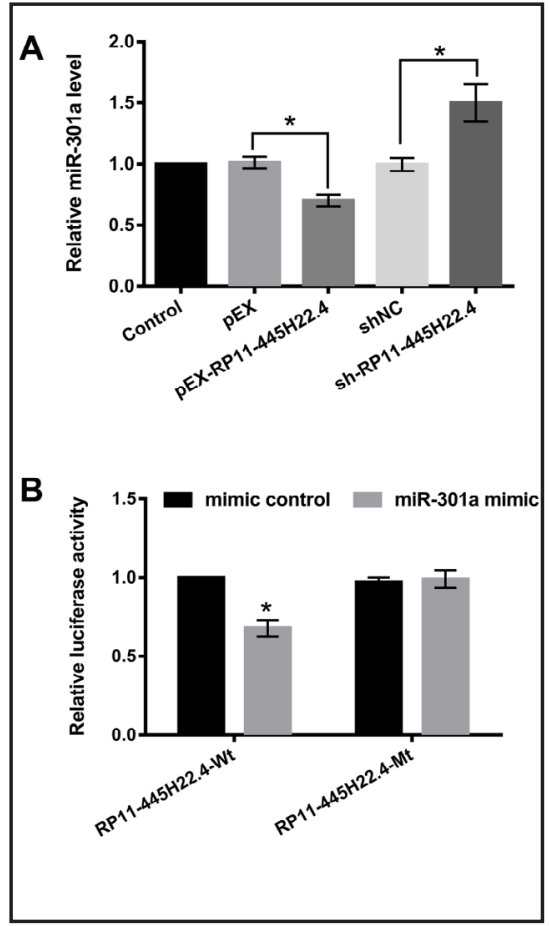

\section{KARGER}


results showed that RP11-445H22.4 inhibition enhanced cell viability, reduced apoptosis after treatment of LPS. Consistently, RP11-445H22.4 inhibition increased Bcl-2 expression and decreased Bax, cleaved-cadpase- 3 and cleaved-cadpase- 9 expressions in LPS-induced cells (Fig. 2C-2E, $P<0.05$ ). The inverse results showed by RP11-445H22.4 overexpression. In addition, RP11-445H22.4 inhibition notably suppressed the expressions and concentrations of IL-1 $\beta$, IL-6, IL-8 and TNF- $\alpha$ after induced by LPS $(P<0.05$ or $P<0.01)$. In a word, inhibition of RP11-445H22.4 exhibited a protective effect on LPS-induced cell injuries in ATGC5 cells.

LncRNA RP11-445H22.4 acted as a molecular sponge of miR-301a

To explore the relationship between IncRNA RP11-445H22.4 and miR-301a, pEX-RP11445H22.4 and sh-RP11-445H22.4 were transfected into ATGC5 cells. The results showed in Fig. 3A, miR-301a expression was obviously inhibited by RP11-445H22.4 overexpression but promoted by RP11-445H22.4 silencing compared with their corresponding controls $(P<$ 0.05). To further confirmation, the luciferase reporters containing RNA RP11-445H22.4 was constructed, which contains Wild-type (Wt) and Mutated-type (Mt) miR-301a binding sites for target investigation. Results showed that the binding RNA RP11-445H22.4-Wt construct suppressed miR-301a overexpression, while RNA RP11-445H22.4-Mt was no distinctly change, indicating that miR-301a directly bound to RP11-445H22.4 (Fig. 3B, $P<0.05$ ). These data suggested that IncRNA RP11-445H22.4 acted as a molecular sponge of miR-301a and negatively regulated miR-301 expression.

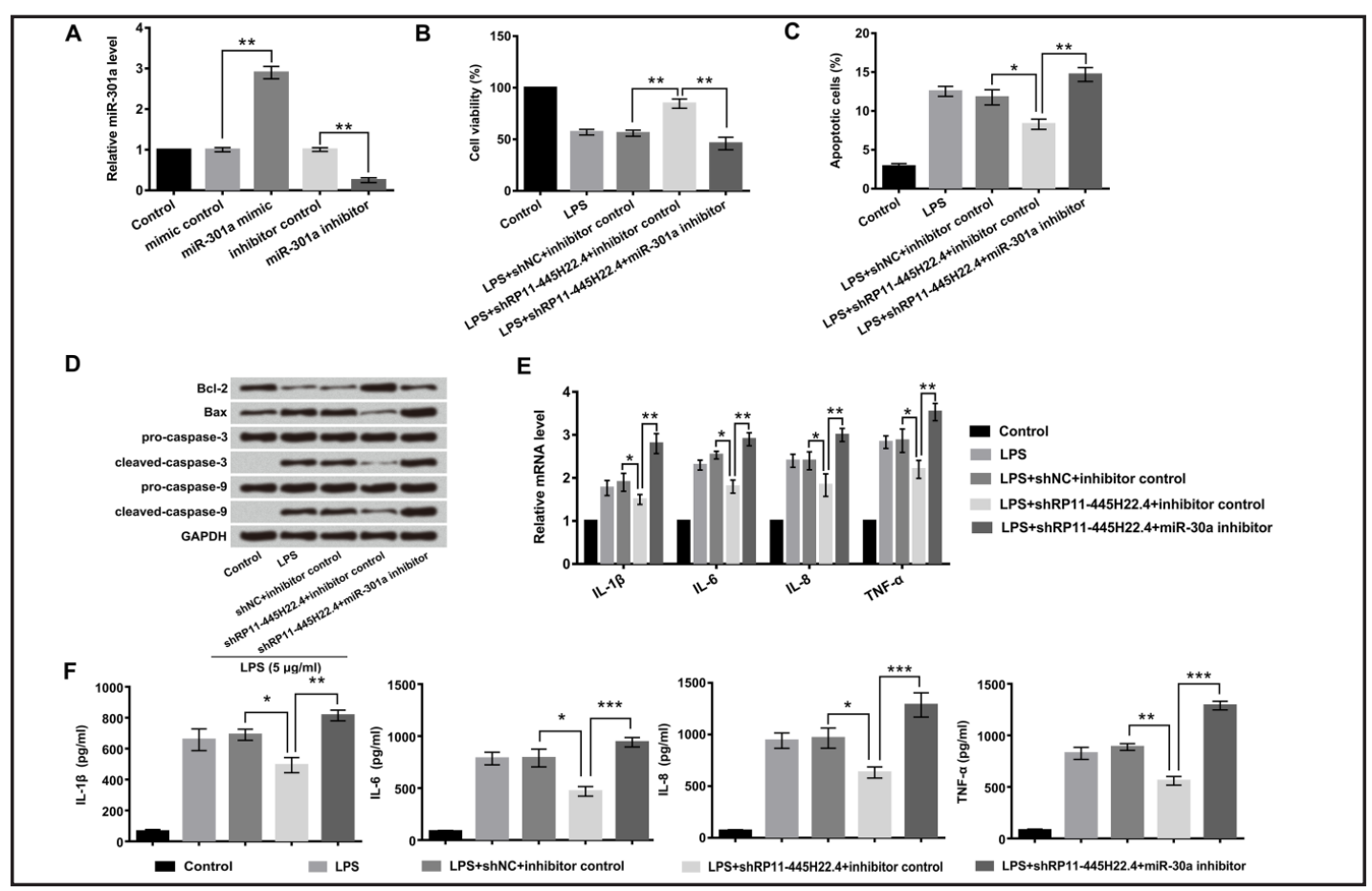

Fig. 4. MiR-301a suppression abolished the protective functions of RP11-445H22.4 silencing on LPS-treated ATGC5 cells. MiR-301a mimic, miR-301a inhibitor, co-transfected with sh-RP11-445H22.4 and miR-301a inhibitor and corresponding controls were transfected into ATGC5 cells. (A) The expression level of miR301a was detected by RT-qPCR. After stimulation with $5 \mu \mathrm{g} / \mathrm{ml}$ LPS, (B) cell viability (C) cell apoptosis (D) protein levels of apoptosis-related factors (E) the mRNA of inflammatory cytokines (F) the concentration of inflammatory cytokines were detected by CCK-8, flow cytometry, western blot, RT-qPCR and ELISA, respectively. LncRNA: long non-coding RNA; miR-301a: microRNA-301a; LPS: lipopolysaccharide; RT-qPCR: realtime quantitative PCR; CCK-8: Cell Counting Kit-8; ELISA: enzyme linked immunosorbent assay; ${ }^{*}, \mathrm{P}<0.05$, $* *, \mathrm{P}<0.01, * * *, \mathrm{P}<0.001$. 
MiR-301a suppression abolished the protective functions of RP11445H22.4 silencing on LPS-treated ATGC5 cells

Further, we investigated whether miR-301a was involved in IncRNA RP11$445 \mathrm{H} 22.4$ regulated LPS-induced cell injuries in ATGC5 cells. To verify this suppose, ATGC5 cells were transfected with miR-301a mimic, inhibitor, sh-RP11445H22.4 and corresponding controls. As demonstrated in Fig. 4A, the expression of miR-301a was visibly increased by miR301a mimic, while decreased by miR301a inhibitor $(P<0.01)$. Co-transfected with sh-RP11-445H22.4 and miR-301a inhibitor suppressed LPS-induced cell viability, meanwhile promoted apoptosis compared with sh-RP11-445H22.4 group (Fig. 4B and 4C, $P<0.05$ or $P<0.01$ ). Western blot results showed that cotransfected with sh-RP11-445H22.4 and miR-301a inhibitor down-regulated Bcl2, up-regulated Bax cleaved-cadpase-3 and cleaved-cadpase-9 expressions (Fig. 4D). In terms of inflammatory cytokines, the expressions and concentrations of IL$1 \beta$, IL-6, IL- 8 and TNF- $\alpha$ were significantly increased by sh-RP11-445H22.4 together with miR-301a inhibitor compared with its control (Fig. 4E and 4F, $P<0.05, P<$ 0.01 or $P<0.001$ ). In sum, these data

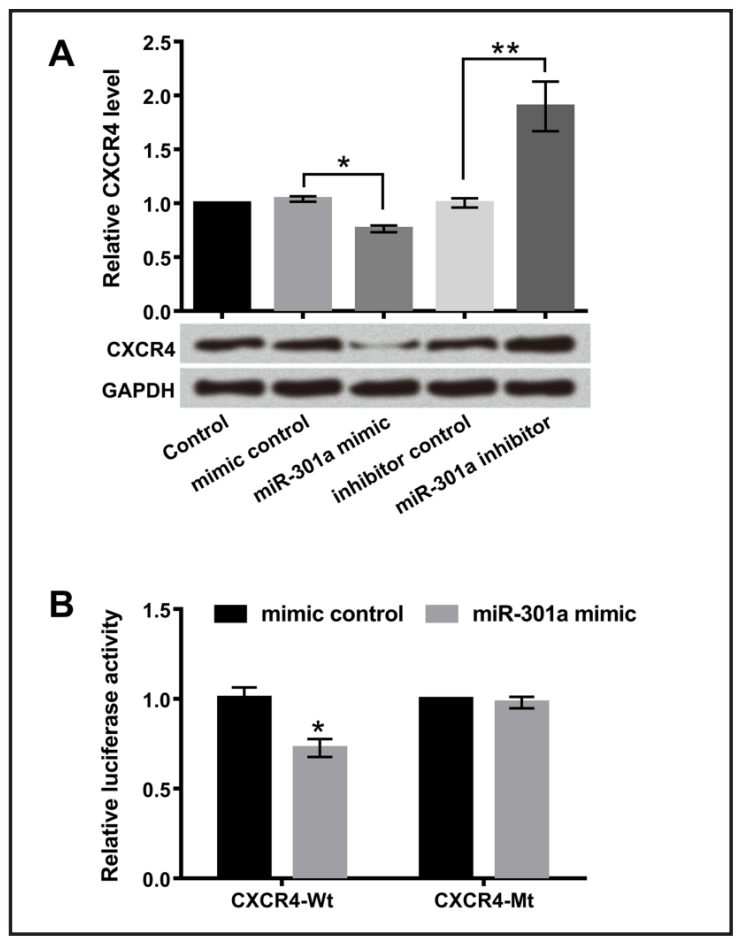

Fig. 5. CXCR4 was a target of miR-301a. ATGC5 cells were transfected with miR-301a mimic, miR-301a inhibitor and mimic or inhibitor controls. (A) The mRNA and protein levels of CXCR4 were measured by RTqPCR and western blot; (B) The activity of a luciferase reporter fused to CXCR4-Wt and CXCR4-Mt were examined by dual-luciferase assay. CXCR4: C-X-C chemokine receptor type 4; miR-301a: microRNA-301a; RT-qPCR: real-time quantitative PCR; Wt: Wild-type; Mt: Mutated-type; *, $\mathrm{P}<0.05,{ }^{* *}, \mathrm{P}<0.01$. indicated that miR-301a suppression abolished the protective functions of RP11-445H22.4 silencing on LPS-treated ATGC5 cells.

\section{CXCR4 was a target of miR-301a}

In ATGC5 cells with overexpression of miR-301a, we found a pronounced reduction in CXCR4 mRNA and protein expressions compared with their control. These opposite results explored by miR-301a suppression (Fig. 5A, $P<0.05$ or $P<0.01$ ). Moreover, miR-301a overexpression markedly decreased the activity of a luciferase reporter fused to CXCR4-Wt, while not the activity of a reporter binding to CXCR4-Mt (Fig. 5B, $P<0.05$ ). Taken together, all results indicated that CXCR4 was a direct target of miR-301a, and was negatively regulated by miR-301a.

CXCR4 silencing promoted cell viability, inhibited apoptosis and inflammatory response in LPS-treated ATGC5 cells

To further clarify the effect of CXCR4 on OA, the stable pEX-CXCR4 and sh-CXCR4 ATGC5 cells were established. As displayed in Fig. 6A, CXCR4 overexpression prominently elevated CXCR4 expression whereas CXCR4 silencing reduced CXCR4 expression $(P<0.01)$. Western blot showed consistent changes in CXCR4 protein. Similar to RP11-445H22.4 inhibition, we found that CXCR4 silencing significantly promoted cell viability and inhibited apoptosis in ATGC5 cells (Fig. $6 \mathrm{~B}$ and $6 \mathrm{C}, P<0.05$ ). Moreover, CXCR4 silencing remarkably up-regulated Bcl-2, down-regulated Bax, cleaved-cadpase- 3 and cleaved-cadpase- 9 expressions in LPS- 


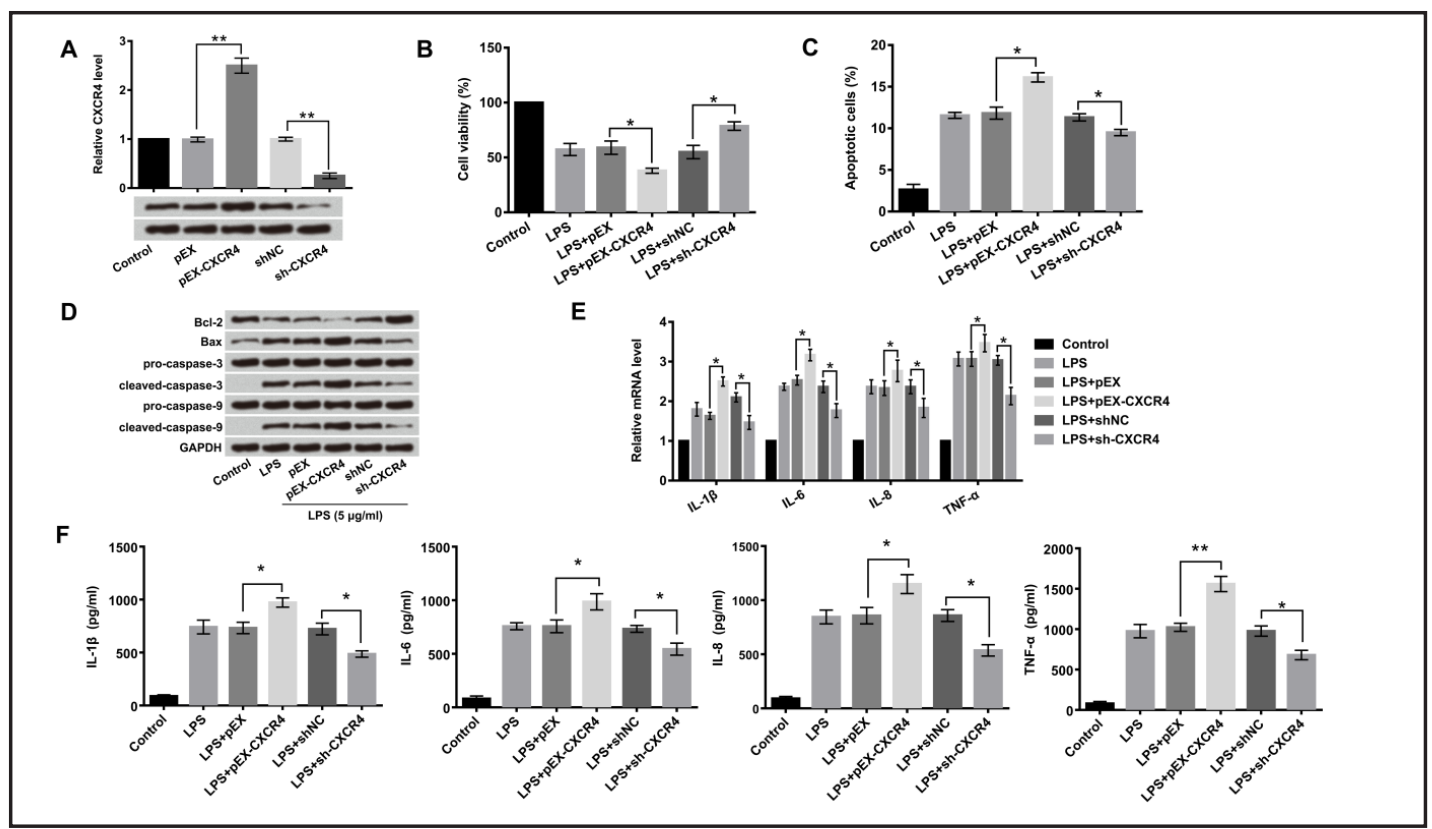

Fig. 6. CXCR4 silencing protected ATGC5 cells against LPS-induced injuries in ATGC5 cells. ATGC5 cells were transfected with pEX-CXCR4 and sh-CXCR4. (A) The mRNA and protein levels of CXCR4 were determined by RT-qPCR. After induction by $5 \mu \mathrm{g} / \mathrm{ml}$ of LPS, (B) cell viability (C) cell apoptosis (D) protein levels of apoptosis-related factors (E) the mRNA of inflammatory cytokines (F) the concentration of inflammatory cytokines were detected by CCK-8, flow cytometry, western blot, RT-qPCR and ELISA, respectively. CXCR4: C-X-C chemokine receptor type 4; RT-qPCR: real-time quantitative PCR; LPS: lipopolysaccharide; CCK-8: Cell Counting Kit-8; ELISA: enzyme linked immunosorbent assay; *, $\mathrm{P}<0.05$, **, $\mathrm{P}<0.01$.

Fig. 7. CXCR4 silencing blocked LPS-activated NF$\kappa \mathrm{B}$ and MAPK/ERK pathways. ATGC5 cells were transfected with pEX-CXCR4, sh-CXCR4 and their controls. After $5 \mu \mathrm{g} / \mathrm{ml}$ of LPS stimulation, (A) the MAPK/ERK pathway factors protein levels and (B) the NF- $\kappa$ B pathway factors protein levels were measured by western blot. GAPDH was used as an internal reference. CXCR4: C-X-C chemokine receptor type 4; LPS: lipopolysaccharide; NF$\kappa \mathrm{B}$ : nuclear factor- $\kappa \mathrm{B}$; MAPK: mitogen-activated protein kinase; ERK: extracellular regulated protein kinases.

induced cells (Fig. 6D). Simultaneously, the mRNA levels of IL-1 $\beta$, IL- 6 , IL- 8 and TNF- $\alpha$ were inhibited by CXCR4 silencing (Fig. 6E, $P<0.05)$. The concentrates of these four inflammatory cytokines were also increased by CXCR4 overexpression (Fig. 6F, $P<0.05$, or $P<0.01)$. The contrary results were confirmed by CXCR4 overexpression. In a word, CXCR4 silencing could promote cell viability, inhibit apoptosis and inflammatory response in LPS-treated ATGC5 cells.

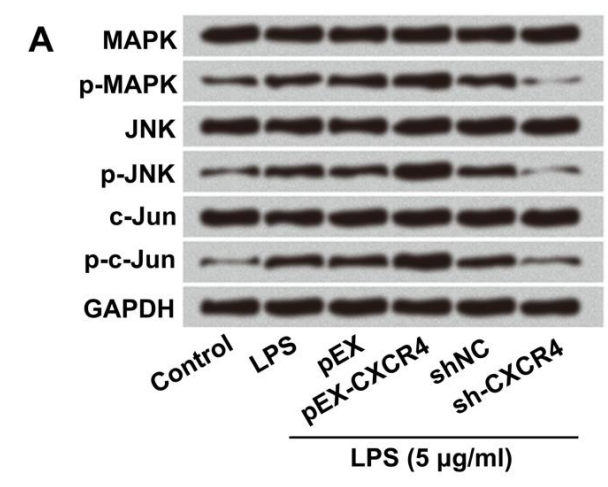

B

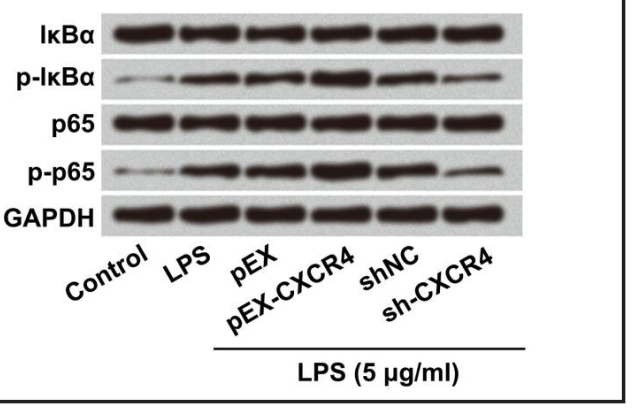




\section{Cellular Physiology Cell Physiol Biochem 2018;45:832-843

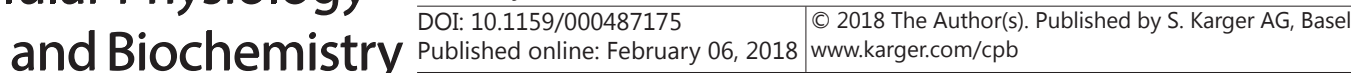 \\ Sun et al.: Role of LncRNA RP11-445H22.4 in OA}

CXCR4 silencing blocked LPS-activated NF- $\kappa B$ and MAPK/ERK pathways

Several studies confirmed that CXCR4 regulated intracellular signaling through some divergent pathways, including NF- $\kappa B$, MAPK/ERK pathways $[15,16]$. Based on the pathway analysis results, we examined the effect of CXCR4 on NF- $\mathrm{BB}$ and MAPK/ERK pathways in ATGC5 cells. Cells were transfected with pEX-CXCR4, sh-CXCR4 and their controls, and the main protein levels of NF- $\kappa B$ and MAPK/ERK pathways were detected by western blot. As results showed in Fig. 7A and 7B, the protein levels of p-MARK, p-JNK, p-c-Jun, p-IкB $\alpha$ and p-p65 were significantly blocked by CXCR4 silencing. However, CXCR4 overexpression activated these five factors expressions. The expressions of MARK, JNK, c-Jun, IкB $\alpha$ and p65 have no obviously changes. Thus, these data indicated that CXCR4 silencing blocked LPSactivated NF- $\kappa B$ and MAPK/ERK pathways in ATGC5 cells.

\section{Discussion}

In present study, we found that LPS reduced cell viability, increased apoptosis and stimulated release IL-1 $\beta$, IL-6, IL-8 and TNF- $\alpha$. However, lncRNA RP11-445H22.4 inhibition significantly recued LPS-induced cell injuries by promoting cell viability, suppressing apoptosis and inflammatory cytokines secretions in ATDC5 cells. In addition, miR-301a directly bound to RP11-445H22.4, and suppression of miR-301a inversed the effects of RP11-445H22.4 inhibition. Furthermore, we confirmed that CXCR4 was a direct target of miR-301a, and CXCR4 silencing exhibited the similarly protective effects of RP11-445H22.4 inhibition on LPS-induced cell injuries in ATDC5 cells. Besides, we found that CXCR4 silencing blocked LPS-activated NF- $\kappa$ B and MAPK/ERK pathways.

As is known to all, LPS plays an important role in bacterial infection and disease evolution [17]. Moreover, LPS acts as endotoxin involving in induction of strong inflammatory responses $[18,19]$. As Li et al. [20] reported that LPS induced gastric cancer cell proliferation, inhibited apoptosis and increased the secretion of IL- $1 \beta$, IL-6, IL-12 and TNF- $\alpha$. Furthermore, Wang et al. [21] confirmed that LPS could induce pro-inflammatory cytokines of IL-1 $\beta$, IL-6, IL-8 and TNF- $\alpha$ productions in macrophage. Similar with previous research, our study showed that LPS significantly decreased cell viability, and increased apoptosis and induced IL-1 $\beta$, IL-6, IL-8 and TNF- $\alpha$ secretion. Overall, these data indicated that LPS could cause cell injuries in ATDC5 cells.

Several studies demonstrated that abnormal expression of lncRNA RP11-445H22.4 play an important role in biological processes and might be involved in variety kinds of cancers or diseases [22]. As Steck et al. [23] revealed that IncRNA H19 acted as a key regulator in chondrocyte metabolism process in OA. Xing et al. [14] reported that lncRNA RP11-445H22.4 was up-regulated in OA compared with normal cartilage tissues. Based on these previous studies, we further explored the effects of IncRNA RP11-445H22.4 on LPSinduced cell injuries in OA. Our results demonstrated that lncRNA RP11-445H22.4 inhibition as a protector rescued LPS-induced cell injuries in ATGC5 cells, as promoted cell viability, inhibited apoptosis and reduced inflammatory cytokines secretions.

Recently, miRNAs have been displayed in various research fields to have an important regulatory role [24, 25]. In terms of miR-301a, abnormal expression of miR-301a has been reported in various human diseases, such as breast cancer, hepatocellular carcinoma (HCC) and colorectal cancer (CRC) [26]. Moreover, accumulating evidences indicate that miR-301a has significant influence in diverse range of cellular processes, including cell proliferation, apoptosis, metastasis and immune response [27, 28]. As Liang et al. [29] displayed that miR-301a promoted cell proliferation and reduced apoptosis in multiple myeloma (MM). Furthermore, Huang et al. [28] demonstrated that down-regulated of miR-301a could inhibit pro-inflammatory cytokines in toll-like receptor-triggered macrophages. However, whether miR-301a was involved in LPS-induced cell injuries in OA remain not investigated. In present study, we confirmed that IncRNA RP11-445H22.4 acted as a molecular sponge of miR-301a, and miR-301a suppression significantly abolished the protective functions of 
RP11-445H22.4 inhibition against LPS-induced injuries. Above all, these data suggested that IncRNA RP11-445H22.4 inhibition alleviated LPS-induced cell injuries by regulation of miR301a in ATGC5 cells.

To further explore the possible effect of miR-301a on LPS-induced cell injuries in OA, a target of miR-301a was confirmed by luciferase reporter assay. We found that CXCR4 was a direct target of miR-301a, and negatively by miR-301a. Recently, CXCR4 has been verified as a direct target of many miRNAs, such as miR-221-3p, miR-9, miR-146a and miR-126 [3033]. Moreover, CXCR4 play an important role in cell growth and development. As Duan et al. [34] demonstrated that CXCR4 was a bona fide target of miR-133b, and CXCR4 silencing promoted cell colonies and apoptosis. Our findings were partly similar with previous study that CXCR4 was a target of miR-301a and CXCR4 as a protector in LPS-induced cell injuries in ATGC5 cells.

NF- $\kappa B$ and MAPK/ERK play vital roles in the inflammatory and immune response [3537]. In terms of $\mathrm{OA}$, the activated NF- $\mathrm{KB}$ molecules trigger the expression of an array of genes which induce destruction of the articular joint, and leading to OA onset and progression [38]. Moreover, inhibition of the p38-MARK signaling pathway suppressed apoptosis and expression of pro-inflammatory cytokines of IL-1,IL-6 and TNF- $\alpha$ in osteoarthritis chondrocytes [39]. Besides, the inhibition of ERK was involved in PLC $\gamma 1$-mediated MMP-13 expression in human OA chondrocytes, with important implication for the understanding of OA pathogenesis [40]. In our study, we explored that CXCR4 silencing blocked LPS-activated NF- $\mathrm{KB}$ and MAPK/ERK pathways in ATGC5 cells, further regulating cell viability, apoptosis and inflammatory injury in $\mathrm{OA}$.

\section{Conclusion}

These results demonstrated that lncRNA RP11-445H22.4 silencing acted as a protector in LPS-induced cell injuries by regulation of miR-301a in ATGC5 cells. Our findings imply that lncRNA RP11-445H22.4 might a serve as a potential therapeutic target in OA.

\section{Acknowledgements}

The work received no funding from any agency.

\section{Disclosure Statement}

There was no conflict of interests.

\section{References}

1 Okubo M, Okada Y: Destruction of the articular cartilage in osteoarthritis. Clinical Calcium 2013;23:17051713.

2 Arden N, Blanco F, Cooper C, Guermazi A, Hayashi D, Hunter D, Javaid MK, Rannou F, Roemer F, Reginster JY: Atlas of osteoarthritis. Springer Healthcare Ltd 2014; 2014:69-82.

3 Hsiao YH, Chiu YM, Tsai HD, Chou MC, Yeh GP: Clinical Applications of Ultrasound in Osteoarthritis. Changhua J Med 2016;14:107-114.

4 Lane NE, Shidara K, Wise BL: Osteoarthritis year in review 2016: clinical. Osteoarthritis Cartilage 2017;25:209-215.

5 Schnitzer TJ, Ekman EF, Spierings ELH, Greenberg HS, Smith MD, Brown MT, West CR, Verburg KM: Efficacy and safety of tanezumab monotherapy or combined with non-steroidal anti-inflammatory drugs in the treatment of knee or hip osteoarthritis pain. Ann Rheum Dis 2015;74:1202-1211. 


\section{Cellular Physiology Cell Physiol Biochem 2018;45:832-843 and Biochemistry Published \begin{tabular}{l|l} 
DOI: $1159 / 000487175$ & 2018 The Author(s). Published by S. Karger AG, Basel \\
www.karger.com/cpb
\end{tabular}

6 Paschos NK: Recent advances and future directions in the management of knee osteoarthritis:Can biological joint reconstruction replace joint arthroplasty and when? World J Orthop 2015;6:655-659.

7 Angrand PO, Vennin C, Le BX, Adriaenssens E: The role of long non-coding RNAs in genome formatting and expression. Front Genet 2015;6:165.

8 Chen WX, Zhang ZG, Ding ZY, Liang HF, Song J, Tan XL, Wu JJ, Li GZ, Zeng Z, Zhang BX: MicroRNA-630 suppresses tumor metastasis through the TGF- $\beta$ - miR-630-Slug signaling pathway and correlates inversely with poor prognosis in hepatocellular carcinoma. Oncotarget 2016;7:22674-22686.

-9 Pearson MJ, Jones SW: Long non - coding RNAs in the regulation of inflammatory pathways in rheumatoid arthritis and osteoarthritis. Arthritis Rheumatol 2016;68:2575-2583.

10 Huang RS, Hu GQ, Lin B, Lin ZY, Sun CC: MicroRNA-155 silencing enhances inflammatory response and lipid uptake in oxidized low-density lipoprotein-stimulated human THP-1 macrophages. J.Investig Med 2010;58:961-967.

11 Liu Q, Zhang X, Dai L, Hu X, Zhu J, Li L, Zhou C, Ao Y: Long Noncoding RNA Related to Cartilage Injury Promotes Chondrocyte Extracellular Matrix Degradation in Osteoarthritis. Arthritis rheumatol 2014;66:969-978.

12 Zhang G, Wu Y, Xu D, Yan X: Long Noncoding RNA UFC1 Promotes Proliferation of Chondrocyte in Osteoarthritis by Acting as a Sponge for miR-34a. DNA and cell biol 2016;35:691-695.

13 Nan X, Fei C, Wang F, Xun L, Xu W, Lv M, Cheng L: Clinical significance of high expression of circulating serum IncRNA RP11-445H22.4 in breast cancer patients: a Chinese population-based study. Tumor Biol 2015;36:7659-7665.

14 Xing D, Liang JQ, Li Y, Lu J, Jia HB, Xu LY, Ma XL: Identification of long noncoding RNA associated with osteoarthritis in humans. Orthop surg 2014;6:288-293.

15 Lombardi L, Tavano F, Morelli F, Latiano TP, Di SP, Maiello E: Chemokine receptor CXCR4: role in gastrointestinal cancer. Crit Rev Oncol Hematol 2013;88:696-705.

-16 Singh AP, Arora S, Bhardwaj A, Srivastava SK, Kadakia MP, Wang B, Grizzle WE, Owen LB, Singh S: CXCL12/ CXCR4 signaling axis induces SHH expression in pancreatic cancer cells via ERK- and Akt- mediated activation of NF-кB: implications for bidirectional tumor-stromal interactions. J Biol Chem2012;55: 3911539124.

17 Maldonado RF, Sácorreia I, Valvano MA: Lipopolysaccharide modification in Gram-negative bacteria during chronic infection. Fems Microbiol Rev 2016;40:480-493.

18 Wilkins R, Tucci M, Benghuzzi H: Role of plant-derived antioxidants on NF-kb expression in LPS-stimulated macrophages - biomed 2011. Biomed Sci Instrum 2011;47:222-227.

19 Siemensma AD, De Vries YP, Feitsma AL, Garcia PML, Albert VDP: DAIRY BASED COMPOSITIONS WITH LOW LPS, WO, 2013,

-20 Li K, Dan Z, Nie Y, Hu X, Gesang L, Bianba Z, Ze Y, Ciren C: CD14 knockdown reduces lipopolysaccharideinduced cell viability and expression of inflammation-associated genes in gastric cancer cells in vitro and in nude mouse xenografts. Mol Med Rep 2015;12:4332-4339.

-21 Wang M, Zheng W, Zhu X, Xu J, Cai B, Zhang Y, Zheng F, Zhou L, Yang Z, Zhang X, Wang C, Nie S, Zhu J: A Human Anti-Toll Like Receptor 4 Fab Fragment Inhibits Lipopolysaccharide-Induced Pro-Inflammatory Cytokines Production in Macrophages. PLoS ONE 2016;11:e0146856.

-22 Xu N, Wang F, Lv M, Cheng L: Microarray expression profile analysis of long non-coding RNAs in human breast cancer: A study of Chinese women. Biomed Pharmacother 2010;69:221-227.

23 Steck E, Boeuf S, Gabler J, Werth N, Schnatzer P, Diederichs S, Richter W: Regulation of H19 and its encoded microRNA-675 in osteoarthritis and under anabolic and catabolic in vitro conditions. J Mol Med 2012;90:1185-1195.

24 Zhang F, Wang J, Chu J, Yang C, Xiao H, Zhao C, Sun Z, Gao X, Chen G, Han Z: MicroRNA-146a Induced by Hypoxia Promotes Chondrocyte Autophagy through Bcl-2. Cell Physiol Biochem 2015;37:1442-1453.

25 Chen Z, Jin T, Lu Y: AntimiR-30b Inhibits TNF- $\alpha$ Mediated Apoptosis and Attenuated Cartilage Degradation through Enhancing Autophagy. Cell Physiol Biochem 2016;40:883-894.

26 Fang Y, Sun B, Xiang J, Chen Z: MiR-301a promotes colorectal cancer cell growth and invasion by directly targeting SOCS6. Cell Physiol Biochem 2015;35:227-236.

27 Wang M, Li C, Yu B, Su L, Li J, Ju J, Yu Y, Gu Q, Zhu Z, Liu B: Overexpressed miR-301a promotes cell proliferation and invasion by targeting RUNX3 in gastric cancer. J Gastroenterol 2013;48:1023-1033. 


\section{Cellular Physiology Cell Physiol Biochem 2018;45:832-843

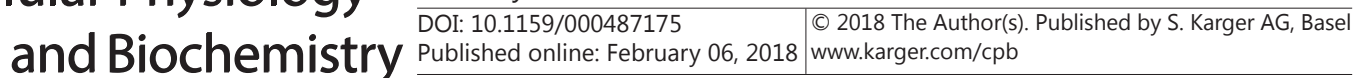 \\ Sun et al.: Role of LncRNA RP11-445H22.4 in OA}

28 Huang L, Liu Y, Wang L, Chen R, Ge W, Lin Z, Zhang Y, Liu S, Shan Y, Lin Q, Jiang M: Down-regulation of miR301a suppresses pro-inflammatory cytokines in Toll-like receptor-triggered macrophages. Immunology 2013;140:314-322.

-29 Liang B, Yin JJ, Zhan XR: MiR-301a promotes cell proliferation by directly targeting TIMP2 in multiple myeloma. Int J Clin Exp Pathol 2015;8:9168-9174.

-30 Xin Z, Zhao FC, Yong P, Li DY, Yao SC, Sun SS, Guo KJ: Downregulation of miR-221-3p contributes to IL$1 \beta$-induced cartilage degradation by directly targeting the SDF1/CXCR4 signaling pathway. J Mol Med 2017;95:615-627..

-31 Lu J, Luo H, Liu X, Peng Y, Zhang B, Wang L, Xu X, Peng X, Li G, Tian W: miR-9 targets CXCR4 and functions as a potential tumor suppressor in nasopharyngeal carcinoma. Carcinogenesis 2014;35:554-563.

32 Quaranta MT, Olivetta E, Sanchez M, Spinello I, Paolillo R, Arenaccio C, Federico M, Labbaye C: miR-146a controls CXCR4 expression in a pathway that involves PLZF and can be used to inhibit HIV-1 infection of CD4(+) T lymphocytes. Virology 2015;478:27-38.

-33 Zhao Y, Li C, Wang M, Su L, Qu Y, Li J, Yu B, Yan M, Yu Y, Liu B: Decrease of miR-202-3p Expression, a Novel Tumor Suppressor, in Gastric Cancer. PLoS ONE 2013;8:410-414.

-34 Duan FT, Qian F, Fang K, Lin KY, Wang WT, Chen YQ: miR-133b, a muscle-specific microRNA, is a novel prognostic marker that participates in the progression of human colorectal cancer via regulation of CXCR4 expression. Mol Cancer 2013;12:164.

-35 Hu Y, Liu JP, Zhu Y, Lu NH: The Importance of Toll-like Receptors in NF- $\kappa$ B Signaling Pathway Activation by Helicobacter pylori Infection and the Regulators of this Response. Helicobacter 2016;21:428-440.

-36 Lugrin J, Ciarlo E, Santos A, Grandmaison G, Dos SI, Le RD, Roger T: The sirtuin inhibitor cambinol impairs MAPK signaling, inhibits inflammatory and innate immune responses and protects from septic shock. Biochimica Et Biophysica Acta 2013;1833:1498-1510.

-37 Yun CJ, Mi EK, Ju HY, Pu RP, Youn HY, Lee HW, Lee JS: Anti-inflammatory effects of galangin on lipopolysaccharide-activated macrophages via ERK and NF- $\kappa B$ pathway regulation. Immunopharmacology and Immunotoxicology 2014;36:426-432.

-38 Rigoglou S, Papavassiliou AG: The NF- $\kappa$ B signalling pathway in osteoarthritis. Int J Biochem Cell Biol 2013;45:2580-2584.

-39 Sun HY, Hu KZ, Yin ZS: Inhibition of the p38-MAPK signaling pathway suppresses the apoptosis and expression of proinflammatory cytokines in human osteoarthritis chondrocytes. Cytokine 2017;90:135143.

40 Liu Z, Cai H, Zheng X, Zhang B, Xia C: The Involvement of Mutual Inhibition of ERK and mTOR in PLC $\gamma 1$ Mediated MMP-13 Expression in Human Osteoarthritis Chondrocytes. Int J Mol Sci 2015;16:17857-17869. 